\title{
Síndrome de boca ardiente
}

\section{Burning-mouth syndrome}

\author{
Casariego ZJ*
}

\section{RESUMEN}

El "Sindrome de la boca ardiente" comprende "Glosodynia o Glosalgia". Significa dolor de la lengua. "Glosopyrosis", sensación de quemazón. Disestesia o parestesia es una experiencia de malestar dentro de la boca. "Glosodynia" es usado también cuando está involucrada toda la cavidad bucal.

Objetivo. Realizar una revisión del tema y establecer la hipótesis que, este sindrome tendría un fondo psiconeuroinmunoendócrino relevante en el campo de las enfermedades autoinmunes. Promediando datos obtenidos, $80 \%$ de las mujeres sufren o han sufrido alguna vez de Glosodynia. Con un rango de 40 a 81 años de edad, media de 60 años, correspondiendo 2,9\% al masculino y 15,7\% al femenino. Localización: bordes de lengua $46 \%$, dorso de lengua $46 \%$, labios $50 \%$ y paladar $46 \%$. La etiología permanece desconocida.

La revisión está centrada en describir varios aspectos clínicos, inmunológicos, endocrinológicos y neuronales involucrados en la génesis, enfocando principalmente la relación con: la noradrenalina y las fibras amielínicas, los beta receptores, la histamina, la bradiquinina, las prostaglandinas, el eje hipotálamo-hipófisis-suprarrenal (HHS), el factor librador de corticotrofina (CRF) y la adrenocorticotropina (ACTH), las Interleuquinas 1, 6, 8, 10 y 12, el factor de necrosis tumoral alfa (TNFalfa), el factor de agregación plaquetaria (PAF), los adenocorticoides (AGC), los mastocitos, la proteína A1 (AP-1), factores locales y sitémicos, la respuesta celular inmune y una base psicológica alterada.

Conclusiones. Debería realizarse un diagnóstico a través de la actividad del eje hipotálamo-pituitaria-adrenal del stress, investigando valores de la respuesta inmune celular. Bajo desòrdenes psicológicos serios, el paciente debe ser referido a un especialista apropiado.

Palabras clave: Psicoinmunoendocrinología, stress, interleuquinas, respuesta inmune celular.

\section{SUMMARY}

The "Burning-Mouth-Syndrome" comprise "Glosodynia or Glosalgia" that means pain of the tongue. "Glosopyrosis": burning sensation." Dysesthesia or Paresthesia is a disconfortable experience inside the mouth. Glosodynia is used when the entire oral cavity is involved as well.

The objective is to make a rewie about this syndrome and to postulate an hypothesis about etiology and diagnosis. Generally, about $80 \%$ of women suffer or once have suffered from Glosodynia. With a range of 40-81 years old, mean age 60 years old, correspond $2.9 \%$ male and $15.7 \%$ female. Llocalization: borders of tongue $46 \%$, dorsum of tongue $46 \%$, lips $50 \%$ and palate $46 \%$. The etiology remains unknown. The hypothesis establish that this syndrome has a psychoneuroimmunoendocrinology background, relevant in the field of autoimmune diseases. The present review is centered in describing clinical, immumologic, endocrinologycal and neurologyc pathways involved in its genesis, and to focus its relationship with the role of noradrenalin and amyelinic fibers, the Beta receptors, histamine, bradiquinin, prostaglandins, HHS, CRF and ACTH, Il-1, IL-6,IL-8, IL-10,IL-2, TNFalpha, PAF, TGF-B, GHRH, PRL, glucocorticoids, mast cells, NK AP-1, local and systemic factors and an altered psychological base.

* Exprofesora Titular de Patología y Clínica Estomatológica. Profesora Titular de Farmacología y Terapéutica. Investigadora acreditada y Miembro del Comité de Grado Académico de la Carrera de Doctorado en Universidad Nacional de la Plata. Directora de la Carrera de Especialización de Estomatología Clínica en la Universidad Católica Argentina (UCA).Buenos Aires. Docente Invitada de Estomatología en el Servicio de Infectología del Hospital de Agudos Juan A. Fernández, Bs.As. 
Conclusions: Diagnosis could be made through the hypothalamic pituitary-adrenal axis activity by stress, investigating the cellular immune response. Under a serious underlying psychogenic disorder, patient must be referred to an appropriated specialist.

Key words: Psychoneuroimmunoendocrinology, stress, interleukins, cellular immune response.

Fecha de recepción: 17 de julio 2008.

Aceptado para publicación: 21 de julio 2008.

Casariego ZJ. Síndrome de boca ardiente. Av. Odontoestomatol 2009; 25 (4): 193-202.

\section{INTRODUCCIÓN}

Como en otros temas de la Estomatología, la etiología de este síndrome es muy discutida. Al recibir a los pacientes portadores de signos y síntomas, la mayoría de las veces mórbidos, uno se pregunta cuál es el disparador de todo el proceso. O sea, qué es aquello que aprieta el gatillo para que todo suceda.

Recurriendo a la literatura nacional y extranjera, uno se encuentra conque la nomenclatura dada a través de los años es variada. "Glosodynia o glosalgia", describe dolor de la lengua (1) "glosopyrosis" refiere sensación de quemazón, (2) "parestesia o disestesia" es un grado menor y se describe como " no confortable" (3). Cuando el dolor involucra toda la boca se lo denomina "estomatodinia" y "estomatopirosis" si es sensación de quemazón. A un estado de desagrado o no confortable se lo ha llamado "estomato-disestesia" (4). Algunos autores han usado el término Glosodynia para dolor, sensación quemante, más xerostomía y pérdida de gusto- Cuando no se han encontrado o no se han investigado causas neurológicas, sistémicas y /o locales, se lo llama además "idiopática" (5-7).

Existen publicaciones sobre la prevalencia de los grupos etarios. Resumiendo las cifras de distintos trabajos seleccionados desde 1920 (1) has el año 2007 (8), podríamos rescatar que, los hombres presentan un rango de edad de 30 a 59 años con una prevalencia de un 2,9\% aproximadamente y las mujeres de entre 20 a 59 años (sobre todo entre los 40-81 años) con un rango del 15,7\% Existiendo una asociación comprobada de la xerostomía con el SBA y algunos medicamentos, se pueden citar los resultados obtenidos del estudio de 80 pacientes; Treinta y siete con SBA $(92,5 \%)$ eran mujeres y el $7,5 \%$ eran hombres, con una media de edad de $63 \pm 11,8$ años (9).

Publicaciones consultadas de diferentes décadas, siempre mencionan un predominio evidente de las mujeres sobre los hombres, variando entre 3:1 para el sexo masculina y 9:1 para sexo femenino según diversos autores (10-13).

A través de la bibliografía consultada y de la experiencia personal, extendida a través de varias décadas de ejercicio de la profesión, se ha podido observar que los pacientes manifiestan distintos síntomas, ya sea, en los bordes laterales, dorso y punta de lengua con mayor frecuencia y, en labios, paladar, faringe y laringe, menor. Los pacientes portadores de prótesis removibles lo relacionan con las zonas de soporte, tanto superior como inferior, y terminan por no usarlas aunque ello no mejora los síntomas.

Pocas dolencias en la cavidad bucal necesitan de tanta observación e inspección minuciosa. Las manifestaciones principales son: sensación de quemazón o de ardor o de dolor, separadas, alternas o juntas. He oído a una paciente manifestar: "me quema tanto que me duele y me duele tanto que es como si me quemara y, a veces, me llega hasta la garganta".

Considerando que el dolor es una experiencia no placentera, subjetiva, sensorial y emocional, involucra componentes de percepción, de transmisión y de información. 
El paciente, además de consultar con varios profesionales, necesita discriminar, "saber" sobre aquello que le afecta y que se refiere a sus sentidos. La alteración emocional que le provoca perturba negativamente su aspecto afectivo. Al perdurar en el tiempo, le angustia aún más el pensar "hasta cuándo" se padecerá su vida cotidiana, si irá disminuyendo con el tiempo su calidad de vida o, si encontrará solución en otra nueva consulta. Así va visitando a distintos profesionales. Aparecen trastornos centrales y psicológicos como ansiedad, depresión, insomnio, anorexia, y cambios profundos en la función autónoma. A veces se afecta el habla, la deglución y se acompaña de sequedad de mucosas, xerostomía aparente o bien la acompaña un sabor metálico o amargo, que no desaparece al ingerir alimentos.

Mientras que el dolor agudo es de corta duración y es una reacción de defensa natural ante una agresión física, el dolor crónico, de mayor duración, modifica todos los mecanismos de defensa del individuo. Los factores psicológicos y psicosociales parecen jugar un papel muy importante. Ninguno de los exámenes establece cuál es la causa esencial. Con frecuencia, las molestias o dolores se relacionan en su comienzo con la pérdida de un ser querido, conflictos emocionales, familiares, problemas de tipo afectivo que los han conmocionado, víctimas de asaltos, separación, divorcio, y/o estrés laboral. Se han registrado alteraciones psiquiátricas en el $37 \%$ de los pacientes y, Zilli y colaboradores (4), han observado hasta un $70 \%$ de cuadros depresivos en sujetos con disestesias de mucosa bucal. Somacarrera y colaboradores describen un perfil psicológico alterado asociado (14).

\section{OBJETIVOS}

Los objetivos de este trabajo están dirigidos a realizar una revisión de la literatura nacional e internacional para encontrar, aquellos elementos que puedan esclarecer la etiología de este síndrome. Además, sustentar la hipótesis que establece que, el paciente debería estudiarse no solo clínicamente si no además, investigarlo desde el punto de vista de la psiconeuroinmunología endocrinología.

\section{ETIOLOGÍA}

Frente a un paciente portador de SBA uno se pregunta: ¿Cuál podría ser la causa o etiología y el mecanismo para que, en una zona tan inervada por el sistema nervioso periférico, como es la mucosa bucal, este tipo de dolor-ardor-quemazón produzca tal señal capaz de ser apreciada de esa forma y que, trasmitida a sus centros cerebrales, éstos traduzcan y señalicen la información con tal intensidad, teniendo en cuenta que al examen clínico ċno se observa patología alguna en ninguna zona de la mucosa bucal?

\section{ASPECTO NEUROFISIOLÓGICO}

Siguiendo la neurofisiología convencional, se ha establecido que la mucosa bucal y su prolongación en el orofarins se caracteriza por la presencia de cantidad de nociceptores. Según esta disciplina, la percepción del dolor se visualiza como un proceso de tres estadios: la "apreciación" en los tejidos periféricos detectada por los sensores especializados o nociceptores para el dolor; "la transmisión" de la sensación del dolor, desde la periferia hacia el asta dorsal de la médula y, la "inhibición o amplificación" de esa sensación, en los circuitos neuronales locales (espinales) o en los centros cerebrales (15).

Específicamente, en lo que corresponde a los ganglios nerviosos de cara y cuello, las terminaciones amielínicas llegan inclusive, según los antiguos libros de Anatomía de Testut Lartajet, hasta el mismo seno de los epitelios y tejidos subyacentes (16). (Recordemos que la Norepinefrina es el trasmisor más importante de las fibras adrenérgicas simpáticas postganglionares, las cuales emergen de los ganglios simpáticos y llegan al tórax, abdomen, cabeza y cuello por la cadena simpática cervical).

En este síndrome, generalmente la lengua es la más afectada. Los filetes nerviosos correspondientes a la sensibilidad de la lengua se encuentran en gran parte en las papilas, en forma de terminaciones intrapapilares y son extremadamente variables. Forman ricas arborizaciones de tres clases de las cuales unas son destinadas a las papilas propiamente dichas y las otras forman manojos debajo de los capilares 
papilares. Un gran número de fibras pasan la zona papilar y sus terminaciones llegan al seno epitelial y se ubican entre las células. Ellas se dividen en dos grupos: las verticales que llegan justo al seno superficial del epitelio y ahí terminan en granos aislados, semejantes a gotas, y las otras, horizontales, las cuales se ubican más profundamente en el epitelio y terminan en forma de botones. El nervio lingual se anastomosa con el nervio alveolar inferior, con el facial por medio de la cuerda del tímpano, con el hipogloso, con el istmo de las fauces y con el ramo comunicante del ganglio submaxilar. De modo que existe una amplia red de intercomunicaciones con receptores distribuidos por todas las regiones mencionadas.

Se han reconocido en estas zonas receptores del tipo alfa y beta adrenérgicos (alfa 1 y 2 y beta 1 y beta 2), siendo los mastocitos, en las criptas amigdalinas del tejido subepitelial, en las tonsilas y en la mucosa de las fauces, ricos depósitos de receptores Beta 2 Lo mismo, los linfocitos $B$ y $T$. Los linfocitos $B$ de las tonsilas contienen alrededor de 600 receptores beta 2 por célula y los linfocitos $T$ tienen 200. (Es de interés para este tema el receptor Beta 2, debido a su influencia en la vasodilatación muscular, en la estimulación de la adenilciclasa para formar AMPc y también como dilatador, a partir de la molécula intermediaria guanín nucleótido sensitiva para el influjo del $\mathrm{Ca}++$ ).

La estimulación del receptor Beta determina también que las mucosas sean más fluidas, lo cual favorece la aparición de más receptores Beta, escondidos o crípticos, distribuidos en todo el tejido conjuntivo cerca de los capilares mucosos orales. "El aumento de receptores Beta totales de membrana produce aumento del estado de hipersensibilidad" (17).

\section{ASPECTOS NEUROENDOCRINOINMUNOLÓGICOS}

Bajo la inspiración de los continuo adelantos que proporciona la investigación inmunológica en el extranjero y los estudios que realizan la Sociedad Argentina de Investigación Clínica, la Sociedad Argentina de Asma e Inmunología y los centros de Biología Molecular Universitarios de Buenos Aires, Tucumán, Córdoba, Rosario y La Plata, Argentina, más otros de la bibliografía internacional, se establece en esta presentación como hipótesis, la posibilidad de incluir este síndrome entre aquellos que involucra la psiconeuroendocrinoinmunología

Se tratará entonces de presentar datos que demuestren la existencia de un circuito interconectado entre esta áreas, dentro de las cuales, y dada una señal, se podría desencadenar el síndrome.

El eje hipotálamo-hipófisis-suprarrenal (HHS) regula las concentraciones sistémicas de cortisol y adrenalina producido por la glándula suprarrenal a través de la regulación del factor liberador de corticotrofina (CRF) y de la hormona adrenocorticotrófica (ACTH). A su vez la vía neuronal, regida por estímulos provenientes del hipotálamo y del sistema nervioso autónomo, influye sobre las funciones inmunes a través de neurotransmisores y neuropéptidos que interaccionan con los linfocitos $B$, las células dendríticas y los macrófagos.

Aplicando estos conceptos, observamos que durante los últimos años se ha demostrado que las células de los tejidos mucosos y submucosos bucales están interconectadas y tienen receptores para agentes químicos específicos o moléculas llamadas neuropéptidos endógenos, las cuales cumplen funciones en los sistemas, neuroendocrino e inmune. Este "micro ambiente" proporciona péptidos y proteínas que son utilizados como mensajeros intercelulares (19).

Si bien en un momento se creyó que los neuropéptidos eran solamente secretados por el cerebro o la hipófisis, se ha demostrado con gran sorpresa que los linfocitos pueden también producir péptidos similares, diferentes de los del sistema inmune.

Se sustenta que las células natural killer (NK), los macrófagos y las células dendríticas, distribuidas por todos los tejidos mesenquimáticos (incluido el corion de los epitelios bucales) poseen receptores para la ACTH Esta hormona es elaborada regularmente por la Hipófisis.

El factor liberador de corticotrofina (CRF), que como ya se citó, es sintetizado por neuronas de la corteza cerebral y del hipotálamo, estimula la producción de 
ACTH por la hipófisis, aumenta la liberación de corticoesteroides y, localmente, es una molécula esencial para la liberación de opioides endógenos dentro de un tejido inflamado Un neuropéptido al cual hay que prestarle atención es el llamado sustancia $P$. Se encuentra presente en abundancia en el sistema nervioso central (SNC), en el sistema nervioso periférico (SNP), en el sistema nervioso de nociceptores, e interviene en la contracción del músculo liso. Tiene influencia también en la vasodilatación y en la secreción de glándulas exocrinas y endocrinas. Se ha reportado como un mediador destacado en la inflamación, ya que, además, induce la síntesis de citoquinas pro inflamatorias como las interleuquinas-1 (IL-1), IL-6, del factor de necrosis tumoral alfa (TNF alfa) y tiene influencia sobre la producción por los monocitos de la sustancia P (20).

La sustancia $p$ también influye en los fibroblastos (presentes en el tejido subepitelial de las mucosas). Su acción sobre los neutrófilos reviste singular importancia ya que estimula la producción de leucotrienes B4 y del ácido 5 HETE, la fagocitosis de los macrófagos y por consecuencia el estallido respiratorio, la activación de la ciclooxigenasa e induce la degranulación de las células cebadas y la liberación de Histamina (20).

La histamina tiene un efecto de estimulación de las terminaciones nerviosas sensitivas a través de sus receptores $\mathrm{H} 2$, activando también a las células endoteliales, las cuales bajo su acción, segregan las prostaglandinas PGI2. Debido a la rápida metabolización que posee la histamina, se deduce que sus efectos biológicos los realiza normalmente cerca del sitio adonde se segrega a través de sus receptores $\mathrm{H} 1, \mathrm{H} 2$ y H3. A través de su receptor $\mathrm{H} 2$ aumenta el AMPc intracelular y a través del receptor $\mathrm{H3}$, media la segregación de neurotransmisores por el SNC y SNP. Además, estimula a las terminaciones nerviosas periféricas para liberar NA. Se segrega en menos de 300 segundos.

En cuanto a la Bradiquinina: proveniente de la conversión intracelular de la precalicaína pertenece al sistema quininógeno-calicreína-quinina, autocoides vasodilatadores muy potentes que, en concentraciones muy bajas, aumentan el dolor actuando sobre las terminaciones nerviosas. Estimula las células ganglionares, produce descarga de catecolaminas de la médula suprarrenal y provoca generación de prostaglandinas por estimulación de la fosfolipasa A2.

La Bradiquinina actúa en la mayoría de los tejidos a través de los antagonistas de los receptores Beta 2 estimulando a los nociceptores.

Es conocido el papel de las Prostaglandinas en la fisiología del dolor, especialmente las PG1, PG2 y PG3. Los mastocitos producen PGD2 y la estimulación de fibroblastos, de células epiteliales, de condrocitos y de IL-1 entrelazada al TNFalfa, llevan a la producción de PGE2. La activación de células endoteliales con IL-1, bradiquinina o trombina estimula la formación de PGI 2.

La PG2 es hiperálgica, o sea, no produce dolor por sí sola, pero potencia la acción de la Bradiquinina y de todas las demás y aumenta el dolor de estímulos térmicos, mecánicos o químicos. Son importantes en el proceso del dolor porque sensibilizan las terminaciones periféricas de los nociceptores primarios aferentes aumentando la información de dolor a nivel espinal (21).

De lo expuesto hasta ahora y basándonos en nuestra hipótesis, se tendría que investigar (como mínimo) los dosajes de: ACTH plasmática, del factor liberador de corticotrofina (CRF), IL-1, IL-6, TNFalfa, sustancia P, Histamina, PG1, 2 y 3, PGI2 y Bradiquinina.

\section{Interrelación del aspecto psíquico}

Relacionando el aspecto psicológico de estos pacientes y el aspecto neuroendocrino, algunos trabajos recientes enfatizan la relación complicada de estresores internos y la activación del eje HHS que modifican al SI.

El impacto del estrés suele medirse determinando la concentración plasmática de ACTH y/o de los glucocorticoides. Diversos estudios clínicos y preclínicos establecen que no todos los estresores producen los mismos cambios en el SNC y en el sistema endocrino, que es importante el aspecto psicológico 
del paciente y que los efectos del stress no siempre son iguales ya que se genera una cascada de eventos que se prolonga en el tiempo, modulada por las catecolaminas, las endorfinas y los glucocorticoides.

El estrés psicológico puede además estimular también la producción del Factor Transformante Beta (TGF-B), del Interferón gamma INFg e IL-1, citoquinas pro inflamatorias. Los efectos biológicos de IL-1 incluyen la sobre expresión de las moléculas de adhesión (MA), citoquinas, ácido araquidónico, metabolitos ácidos, acumulación de neutrófilos, proliferación de fibroblastos, angiogénesis y síntesis de proteína en la fase aguda. Se siguen realizando estudios al respecto en ratas, tratando en este sentido, de obtener un componente autoinmunológico inducido. La conservación de las funciones e integridad de la proteína A-1 o (activating protein-1) presente después de provocado un estímulo, ha resultado ser sumamente importante, porque interviene en la activación de la transcripción celular de los LT y LB, en la maduración de los timocitos y en procesos de apoptosis. Esta proteína interacciona con el factor de transcripción nuclear NF-kappa B, se sinergia con él y disminuye la actividad de todos los genes nucleares inductores de citoquinas pro inflamatorias tratando d e equilibrar el sistema (22).

Nuevamente, remitiéndonos a completar un estudio inmunológico, tendríamos que investigar además: TGF-B, INF gamma, IL-1, proteína $C$ reactiva, subpoblaciones activadas de $L T$ y $L B$.

\section{Estudio clínico del paciente con SBA}

Desde 1920 a la fecha se ha discutido sobre este síndrome. Las publicaciones de las décadas del 60, 70, 80 y 90 han sido fructíferas. Del año 2000 en adelante, si bien han disminuido en producción, han sido más complejas y productivas.

Con respecto al estudio clínico, se deberá realizar un exhaustivo estudio previo del paciente con "probable" SBA. En la gran mayoría de los casos estaremos frente a una persona difícil, nerviosa, angustiada o problemática. La observación de la fascia es fundamental...
La realización de una exhaustiva Historia Clínica en la primera entrevista, es de primordial importancia y, en donde la anamnesis juega un papel decisivo. Así mismo, una anamnesis médica general y el registro escrito de un interrogatorio detallado sobre los dolores o malestares que el paciente ha padecido o padece, la calidad, intensidad, el aumento o disminución de la sensibilidad al frío o calor, la pérdida del gusto, el aumento al contacto con ciertos alimentos, o la intolerancia a alguno específico son todos datos de sumo interés.

El tema de los medicamentos que el paciente ingiere es de relevancia. Puede estar bajo tratamiento con antimicóticos y/o con antisépticos en forma de buches o colutorios usados en forma constante y diariamente tienen su consecuencia. Puede estar medicada con psicofármacos, con antidepresivos o psicoestimulantes, con antibióticos, con corticoides, con antihipertensivos, anticoagulado, etc.

Cada uno de los medicamentos mencionados contiene drogas base, capaces de producir diferentes huellas en la mucosa bucal. La existencia de distintos metales en sus piezas dentarias o prótesis pueden llevar desde un galvanismo, productor de síntomas desagradables, a reacciones alérgicas. Los estudios de rutina de hemograma y glucosa en plasma y en orina son de valor para detectar algún dismetabolismo.

La descripción por parte de la paciente de su dieta no deberá ser excluida del interrogatorio. El estudio de estrógenos en la mujer y un factor reumatoideo, pueden llevar al especialista a encontrar cofactores importantes. Agregar entonces a nuestra lista dopaje de estrógenos, estudio de hormonas tiroideas ly FR.

\section{Examen de la cavidad bucal}

Indudablemente éste debe realizarlo un estomatólogo.

Se pueden observar cambios en la mucosa bucal que sintetizando pueden referirse a:

- Lesiones primarias y/o secundarias propias de la mucosa bucal. 
- Enfermedades secundarias en la mucosa, de orden o causas sistémicas.

- Infecciones bacterianas, virales o micóticas.

- Enfermedades autoinmunes.

- Reacciones inmunológicas.

- Hipersalina/ hiposalia/ xerostomía.

O, se puede observar una cavidad bucal sana, sin ningún cambio o alteración de la mucosa.

Si es así, estaremos frente a una patología "idiopática". Se considera imprescindible dar la mayor cantidad de razones al paciente, especificando que no se trata de un síndrome "exótico" si no que otras personas lo padecen.

Éste sería el momento de someter a la o al paciente a los estudios recomendados en la Hipótesis aquí expuesta, dando razones por causas, de modo que no parezca extravagante nuestra actitud y sobrecargado nuestro pedido de estudios.

Debido a lo profuso de solicitud de estudios, de análisis y pruebas a los que el paciente tendría que someterse por un lado y costearlos por el otro, se sugiere realizar la investigación por etapas. Si no es posible realizarlo todo de una sola vez. Nuestro objetivo en este punto sería encontrar algún o algunos datos inmunológicos, endocrinos y neuropáticos que podrían llevarnos a un diagnóstico de certeza y a un plan de tratamiento. De todas maneras, se debe solicitar al paciente la consulta a un psicólogo, explicando también el motivo y que sea tomado como parte del tratamiento. La experiencia nos dice que el paciente, sin embargo, requiere la cura mágica. Algo que alivie sus síntomas. No es el propósito de este trabajo la indicación de una terapéutica medicamentosa previsora o empírica, pero la fitoterapia, en este aspecto puede conceder efectos paliativos y darnos tiempo mientras recibimos los resultados de todos los estudios solicitados.

Traduciendo del prólogo que el Dr. Isaac van der Waal escribió en su tratado de SBA (23) extractamos lo siguiente: "El tratamiento del paciente que sufre de SBA requiere paciencia, y comprensión de sus médicos. A través de la utilización de los conocimientos científicos puede ser atendidos con éxito una gran cantidad de pacientes".

\section{DISCUSIÓN}

Existe una perspectiva histórica del concepto de estrés y, coincidiendo con Chrousos y Gold (24) existiría en algunos pacientes, una desregulación de los aspectos bioquímicos, y neurofisiológicos que favorecería la vulnerabilidad a varias enfermedades y procesos inflamatorios crónicos. Cabría sumar los trastornos del sistema inmune y endocrinos. Estos autores incluyen la importancia de la hiperreactividad a los desencadenantes de tipo ansioso debido a la psicología de base, individual. En la hipótesis presentada se podría hablar de una hiperrespuesta sensorial en aquellos individuos con SBA bajo estado de estrés no controlado dada la cantidad numerosa de nociceptores y receptores Beta adrenérgicos que se encuentran en los tejidos bucales. Estos autores analizan en su publicación las interacciones entre $\mathrm{CRH}$ y NA, y el contexto neuroanatómico de estas interacciones, señalando las proyecciones de neuronas secretoras de CHR que envían sus mensajes desde el núcleo lateral paraventricular hacia los sistemas simpáticos y la transmisión al cerebro posterior. Esto explicaría los efectos centrales sobre la conducta, propios de la CRH. Varios autores últimamente (25-29) refieren una elevada frecuencia en la disminución de la sensibilidad en la lengua en SBA para estímulos térmicos, y pérdida del gusto. Una de las conclusiones (25) se basa en estudios histológicos con coloración hematoxilina-eosina, en donde observaron la apariencia de "venas varicosas" de las fibras unitarias nerviosas terminales, pegadas a las paredes de los vasos sanguíneos. Si bien en parte el síndrome de boca ardiente sea la expresión clínica de una polineuropatía de las fibras de pequeño calibre y, que se puede localizar en la lengua, donde existe una mayor densidad de fibras nerviosas sensitivas, las lesiones nerviosas "irritativas", que han detectado estos autores no coincide con la sintomatología de la mayoría de los portadores de SBA. Son sensibles a los cambios térmicos y muchas veces refieren que "calman" su sensibilidad, introduciendo cubos de hielo en la boca o tomando casi constantemente agua fría. La ausencia o defectos en la gustación $(26,27$,) probablemente nos esté llamando la atención a diferentes aspectos o variables en estos pacientes. La disgeusia controlable con ácido alfa tióctico (Femiano, Gombos E Scully (28) y Femiano, Scully, Deems, et al (29.) no supondría 
entonces analgesia ni hiperalgesia, propias de algunos otros individuos.

El protocolo general de manejo clínico de los pacientes con SBA publicado por Donat y Serrano Martínez (30) en donde se enumeran distintos mecanismos terapéuticos, para tratar anemias, corregir glucemias, evitar ciertos fármacos, y tratamientos orales, es correcto para aquellos pacientes en los cuales los análisis clínicos de rutina y odontológicos presentan valores anormales. Si bien no deben obviarse, los estudios recomendados en la hipótesis del presente trabajo completarían una investigación más exhaustiva en orden de llegar finalmente a la etiopatogenia del síndrome.

Se recomienda así mismo la lectura de: "Las conclusiones del Simposium de la Sociedad Española de Medicina Oral sobre "Xerostomía. Síndrome de Boca Seca. Boca Ardiente", llevado a cabo el 23 de febrero del 2007. En el mismo se subrayaron los resultados de un estudio realizado en forma de encuesta extraída de artículos especializados en boca seca, en 152 oficinas de farmacias y sobre un total de 563 cuestionarios recogidos. El objetivo era evaluar el grado de Xerostomía o Síndrome de Boca Seca y las consecuencias de la xerostomía y la hiposalia en Ginecología, en Odontología, en Medicina Interna, en Oncología, en patologías glandulares y en Psiquiatría (8). El artículo sobre la asociación del síndrome de boca ardiente con xerostomía y medicamentos (9) señala que, una xerostomía de tipo subjetivo, fue referida por el $75 \%$ de 37 pacientes con SBA y el $45 \%$ del grupo control. El flujo salival estimulado entre los pacientes con SBA presentó una media de 1,25 00,67 ml/min. El 90\% de los pacientes presentaron flujo normal y el $10 \%$ hiposalivación. La observación de nuestros pacientes refleja cifras similares. Coincidimos evidentemente que los factores psicológicos desempeñan un importante papel como cofactores etiológicos, agravados por la ingesta de medicamentos psicotropos, en el afán del paciente de luchar contra la ansiedad y/o la depresión”. Los analgésicos, psicotropos, fármacos para el aparato digestivo y cardiovascular, antihipertensivos, específicamente inhibidores de la conversión de la angiotensina (IECA) se han identificado como "protectores del SBA". (30) Teniendo en cuenta el perfil psicológico, este paciente que varía entre de- presión y estrés crónico, la teoría monoaminérgica de la de la primera propone que un déficit de noradrenalina (NA) y /o de serotonina (5-HT) centrales podría estar relacionado con esta enfermedad (14). Por otro lado, una segunda teoría sustenta la desregulación del sistema Hipotálamo-Hipófisis-Suprarrenal (HHS) con la consecuente hipersecreción del CRF, ACTh y cortisol.. La tercera hipótesis publicada refiere la participación clave de las citoquinas, las cuales determinarían una hipersecreción importante de la ACTH, CRF, prolactina y cortisol. (34). Los glucorticoides (GC) endógenos son potentes reguladores negativos de la síntesis y liberación de CRF a través de los receptores localizados en el eje HHS. (36). Algunas de las manifestaciones descritas en individuos con episodios recientes de muertes de familiares, de parejas o amigos incluyen supresión a la respuesta de linfocitos, a la estimulación mitógena (PHA), reducción de la actividad de las células killer (NK) y alteración del ratio CD4/CD (30).

\section{CONCLUSIÓN}

Nos lleva a la reflexión la lectura de las publicaciones realizadas sobre las interrelaciones o llamadas "vías bidireccionales" del eje hipotálamo, hipofisario, suprarrenal, sus relaciones con el sistema nervioso autónomo y periférico, y su influencia en los cambios inmunitarios que se producen en pacientes bajo experiencias de estrés y susceptibilidad individual. Reconocemos ciertos aspectos de variabilidad en La descripción del síndrome.

Aspiramos, con la investigación de los principales indicadores de todo este macrosistema, en nuestra cohorte de pacientes portadores de SBA, llegar a conclusiones definitivas, darlas a conocer y que demuestren la hipótesis expuesta.

\section{BIBLIOGRAFÍA}

1. Ziskinde, Moutorn R. Glosodynia; a study idiopática or lingual pain. J Am Dent Assoc. 1946;33: 1422-32.

2. Engman MF. Burning Tongue. Arch Dermatology Syphlil 1920;6:137-8. 
3. Locker D, Gresham M: Prevalencia facial disonfort. Preliminary results of a mail survey. Community. Dent Oral Epiddemiol 1987;15:169-72.

4. Zilli C, Brooke RL, Lan CL. Screening for psychiatric illnes patients with dysesthesis by means of the GHQ.

5. Gilpin SF. Glosodynia. JM. MED Assoc.1936; 106:1722-4.

6. Poire M: Etude clinque et therapeutique de glosodynes.'A propos de 130 cas. These doctorale.Universitè Pierre et Marie Curie. Parìs VI;1981:224-7.

7. Glick D, Ben-Argeh H, Gutman D, Szargel R. Relation between idiophatic glosodynia and salivary flow-rate and coment. Int $\mathrm{J}$ Oral Surg 1976;5:161-5.

8. Bascones A, Tenovuo J, Ship J, Turner M, MacVeigh I, Lopez-lbor JM, et al. Conclusiones del Simposium 2007 de la Sociedad española de Medicina Oral sobre "Xerostomía. Síndrome de Boca Seca. Boca Ardiente". Avances en Odontoestomatología/3. Documento Simposium sobre xerostomía. Madrid, 23 de febrero 2007.

9. Marques Soares MS, Chimenos Küstner E, Subirá Pifarre C, Rodriguez De Rivera Campillo E, López J. Asociación de sindrome de boca ardiente con xerostomía y medicamentos. MED Oral Pathol Oral Cir. Bucal 2005;10:301-8.

10. Wagner IV. Möglichkeiten der Objektivierung des syndroms des glossalgic und stomatodynie. 1Mitteilun: Quantitative Speilchelmessungen. Zahn Mund und Kieferheilkd. 1984;72:211-6.

11. Karshan M, Kusc AH, Silvers HF, Stein G, Ziskin DE. Studies in the etiology of idiopathic orolingual paresthesis. Amer Jour Dig Dis 1952;19:341-4.

12. Rojo L, Silvestre FJ, Bagán JV, De Vicente T. Prevalence of psychopathology in burning mouth syndrome. Oral Surg Oral Med Oral Pathol 1994; 78:312-6.
13. Tarkkila L, Linna M, Tiitinen A, Lindqvist C, Meurman JH. Oral symptoms at menopause-the role of hormone replacement therapy. Oral Surg Oral Med Oral Pathol Oral Radiol Endod 2001; 92:276-80.

14. Somacarrera ML, Pinos HP, Hernández G, Lucas ML. Síndrome de boca ardiente. Aspectos clínicos y perfil psicológico asociado. Arch Odontoestomatol 1998; 14:299-306.

15. John N Walton.Neurología, estructura y función del Sistema nervioso. En: Fisio Patología. Smith LH, Thier SO. Panamericana ed. Buenos Aires 1983;1001-12.

16. L. Testut D 'Anatomie Humaine. Systeme Nerveux Pèripherique. $8^{a}$ ed. Paris: Octave Doineditur 1905; 381-4.

17. Page CP, Curtis MJ Sutter MC, Allfer MJ Hoffman BB. Fármacos y sistema nervioso central. En: Farmacología Integrada. Kernroin R, Naylos R, Travis MJ, Simone O, Moore PK.HAUCORT ediciones, Madrid 1999 p. 93-104.

18. Heim MH. The JAK-STAT pathway: cytokine signalling from the receptor to the nucleus. J Recept Signal Transduct Res 1999;19:75.

19. Abbas AK, Lichtman AH. Inmunología celular y molecular. Elservier editores $5^{\mathrm{a}}$ ed, Madrid: Elservier Imprint 2004;163-93.

20. Silvia G Correa. Neuropéptidos: moduladores endógenos de la función inmune. En: Inmunopatología molecular: nuevas fronteras de la Medicina. Gabriel Adrián Rabinovich, Panamericana ed, Buenos Aires 2004. p. 559-63.

21. Fink S, Sasiain MC, Finiasz M, de la Barrera S, Bottaso O. Aspectos moleculares de las citoquinas y sus receptores. En: Inmunopatología molecular: nuevas fronteras de la medicina. Gabriel Adrián Rabinovich, Panamericana ed, Buenos Aires 2004. p. 208-13.

22. Mormede P, Dantzer R, Michaud B, Kelly KW, Le Moal M: Influence of stressor predictability and 
behavioural control on lymphocyte reactivity, antibody response and neuroendocrine reactivation in rats. Physiol Behav 1988;43: 577.

23. Isaac van der Waal. Mundschleimhautbrennen. Das Burning-Mouth Syndrom. Deutscher ÄrzteVerlag. Köln 1992: p. 9.

24. Chrousos GP, Gold PW. Concepto de estrés (tensión) y alteraciones en los sistemas de estrés. JAMA 1993;2(9):324-33.

25. Lauritano D, Calzavara D, Papagna R, Baldoni M, Bascones A. Evidencia del sindrome neuropático en un estudio neurofisiológico e inmunohistoquímico de las fibras nerviosas en pacientes con sindrome de boca ardiente. Advances en Odontoestomatología, 2003;19:2:82-94.

26. Grushka M, Epstein JB, Gorsky M. Burning mouth syndrome and other oral sensory disorders: a unifying hypothesis. Pain Rees Manag 2003; 8:133-5.
27. For marker BK, Frank ME, taste function in patients with oral burning. Chem. Senses 2000; 25:575-81.

28. Femiano F, Gombos F, Esposito V, Scully C. Burning Mouth Syndrome (BMS): evaluation of thyroid and test. Med oral pathol oral cir bucal (Internet) Madrid, ene-feb 2006.

29. Femiano F, Scully C. Burning mouth syndrome (BMS): double blindcontrolled study of alphalipoic acid (thioctic acid) therapy. J Oral Pathol med 2002;31:267-9.

30. Silvestre Donat FJ, Serrano Martínez C. Sindrome de boca ardiente: revisión de conceptos y puesta al día. Medicina Oral 19772:30-8.

\section{CORRESPONDENCIA}

Zulema J. Casariego

Bte.Mitre 1371-4M-1036 Bs.As. Argentina Telf.: 0054-11-4372-0444 